\title{
Molecular origin of gibbsite particle aggregation in water
}

\author{
LOUISE J CRISCENTI AND TUAN HO
}

\section{Sandia National Laboratories}

Presenting Author: ljcrisc@sandia.gov

The physical and chemical properties of mineral-fluid interfaces have a critical impact on the precipitation and compaction of minerals in natural and anthropogenic environments. The study of gibbsite $\left(\gamma-\mathrm{Al}(\mathrm{OH})_{3}\right)$ and gibbsite aggregation has significance from a nuclear waste management perspective. Aluminum (oxy)hydroxides such as gibbsite have become the focus of considerable research because some of the Hanford (WA) and Savannah River (SC) tanks contain residual radioactive waste with aggregates of aluminum (oxy)hydroxide minerals. From experiments reported in the literature, a twostage model is used to describe particle aggregation. First, two particles approach but remain separated by solvent layers. Then, an energy barrier associated with the solvent-separated configuration is overcome, and particles jump into contact. Using classical molecular dynamics (MD) simulations, we investigate this two-stage model and the origin of crystal face selectivity when one gibbsite particle attaches to another in pure water. A comparison of the total free energy $\left(\Delta \mathrm{G}_{\mathrm{t}}\right)$ over the surface area $\left(\Delta \mathrm{G}_{\mathrm{t}} / \mathrm{SA}\right)$ of particle-particle attachment indicates that gibbsite particle attachment through edge surfaces, where the edge surfaces are either (100) or (110) crystal faces, is energetically favorable compared to attachment between two basal surfaces (i.e., (001) crystal faces) or between the basal surface of one particle and the edge surface of another. This result suggests that very small gibbsite crystals (i.e., nuclei), for which the edge and basal SAs are roughly equivalent will preferentially attach through edge surfaces helping the crystal grow laterally. However, for larger gibbsite particles, that have more basal than edge SA, the $\Delta G_{t}$ of particle attachment through the basal surfaces is greater than for edge surfaces, indicating that these particles will preferentially aggregate through basal surface attachments. We hypothesize that interparticle hydrogen bonds from surface $-\mathrm{OH}$ groups drive the final step of particle attachment.

Sandia National Laboratories is a multi-mission laboratory managed and operated by National Technology and Engineering Solutions of Sandia, LLC., a wholly owned subsidiary of Honeywell International, Inc., for the U.S. DOE's NNSA under contract DE-NA0003525. This work was supported by the DOE Office of Science, Office of Basic Energy Sciences, Chemical Sciences, Geosciences and Biosciences Division. SAND2021$2215 \mathrm{~A}$. 